Editorial

\title{
Surgery for Spontaneous Intracerebral Hemorrhage: Current Concept
}

\author{
Partha S. Ray ${ }^{1}$ \\ ${ }^{1}$ Department of Neurology and Clinical Neurophysiology, The \\ Walton Centre for Neurology, National Health Service, Liverpool, \\ United Kingdom
}

Indian J Neurosurg 2021;1:1-5.

Spontaneous intracerebral hemorrhage (ICH) into the brain parenchyma and into the ventricles presents as a severe stroke with high mortality, ${ }^{1}$ with uncontrolled hypertension, cerebral amyloid angiopathy, and more recently the anticoagulant-induced cerebral bleeds representing the main risk factors. ${ }^{2-5}$ Unfortunately, interventions to stop hematoma expansion by rapid reduction of blood pressure, the use of recombinant activated factor eight, or the administration of tranexamic acid have not shown improvement in functional outcome. . $^{6-8}$

The theoretical advantages of evacuating the hematoma and preventing the acute effects of the blood products on the surrounding healthy brain tissue are counterbalanced by the risks of reaching out to the location of the bleeds in the deep basal ganglia structures and the thalamus through healthy cerebral tissue and the additional burden of postsurgical complications. ${ }^{9}$

There is the need for emergency lifesaving surgical evacuation of large lobar hemorrhages and hematomas in the posterior fossa to avoid cerebral or brainstem herniation, and in such situations comparison of best medical management with surgical interventions does not lend itself to a randomized clinical trial (RCT) opportunity for an evidence-based assessment.

In about two-thirds of patients, acute hemorrhage into the brain parenchyma results in stoppage of bleeding through disruption and mass effect within the cerebral tissue. In the remaining one-third, hematoma expansion results in midline shift and an adverse outcome. ${ }^{10}$ The best medical management and neuro intensive care with interventions of recombinant activated factor eight reduced hematoma growth but did not decrease mortality or improve functional outcome. The use of tranexamic acid reduced hematoma expansion but did not improve the functional outcome at 90 days. Two

DOI https://doi.org/ $10.1055 / \mathrm{s}-0041-1726865$ ISSN 2277-954X
Address for correspondence Partha S. Ray, MD, DNB, FICP, FRCP, MRCP, CCST, Department of Neurology and Clinical Neurophysiology, The Walton Centre for Neurology, National Health Service, Liverpool, United Kingdom; National Professor of Neurology, India (e-mail: partha_ray@hotmail.com).

large trials of blood pressure lowering-INTERACT- $2^{11}$ and ATACH-II ${ }^{6}-$ demonstrated that maintaining a systolic blood pressure around 120 to $130 \mathrm{~mm} \mathrm{Hg}$ in the first 24 hours might result in improved functional outcome. ${ }^{12}$

Hematoma volume greater than $30 \mathrm{~mL}$ had statistically unfavorable outcome and a volume greater than $60 \mathrm{~mL}$ with Glasgow Coma Scale (GCS) score lower than 8 had greater than $90 \%$ predicted 30 -day mortality. A volume greater than $150 \mathrm{~mL}$ through abrupt increase in intracranial pressure (ICP) and critical reduction of cerebral perfusion pressure (CPP) leads to death. ${ }^{13,14}$ Much smaller hematoma volumes in the posterior fossa due to obvious limitations of space to expand leads to brainstem herniation/compression with hydrocephalus and clinical deterioration when hematoma evacuation is of lifesaving consequence..$^{15,16}$

Additional adverse effects of the blood products from the hematoma and secondary inflammation and edema resulting from the same would compound the mass effect, midline shift and decreasing cerebral perfusion consequent to rising ICP. ${ }^{17,18}$

\section{Availability of Surgical Treatment Alternatives}

Several surgical treatment alternatives are available, as discussed below.

First, insertion of external ventricular drain (EVD) for intraventricular hemorrhage (IVH) management and ICP monitoring (in ICH): IVH occurs in $45 \%$ of patients with ICH, and interfering with normal cerebrospinal fluid (CSF) flow causes acute hydrocephalus and independently predicts an unfavorable outcome. ${ }^{19}$ The urgent placement of an EVD with drainage of CSF and ICP monitoring (target less than $20 \mathrm{~mm}$ $\mathrm{Hg}$ and CPP more than $60 \mathrm{~mm} \mathrm{Hg}$ ) is the goal. ${ }^{20}$ In the CLEAR

(c)2021. Neurological Surgeons' Society of India.

This is an open access article published by Thieme under the terms of the Creative Commons Attribution-NonDerivative-NonCommercial-License, permitting copying and reproduction so long as the original work is given appropriate credit. Contents may not be used for commercial purposes, or adapted, remixed, transformed or built upon. (https://creativecommons.org/licenses/by-nc-nd/4.0/).

Thieme Medical and Scientific Publishers Pvt. Ltd. A-12, 2nd Floor, Sector 2, Noida-201301 UP, India 
III $^{21}$ trial, low-dose intraventricular recombinant tissue plasminogen activator ( $\mathrm{r}$-tPA) was compared with placebo in small spontaneous ICH with volume less than $30 \mathrm{~mL}$ and IVH obstructing the third and fourth ventricles. The targets were: opening of the third and fourth ventricles, the relief of the IVH mass effect, or $80 \%$ clot removal. A favorable outcome was defined as a 6-month modified Rankin scale (mRS) score of 0 to 3; this was not significantly different in the r-tPA and saline groups. In the r-tPA group, $11 \%$ lower case fatality was noted, which balanced against an $8 \%$ increase in patients in a vegetative state. Only a third of patients in the treatment arm had the desired end point of $80 \%$ of intraventricular clot removal. The 6-month functional outcome compared with placebo was no better.

Clot removal by neuro endoscopy in combination with EVD placement in a meta-analysis of 11 studies (out of which only 5 RCTs were included) found that neuro endoscopy with EVD was superior than EVD and tPA in terms of mortality, effective IVH evacuation, favorable functional outcome, and the need for ventricular peritoneal shunt. These preliminary results need to be followed through with further studies including comparisons between EVD and neuro endoscopy. ${ }^{22-24}$

Second, craniotomy for supratentorial hemorrhage drainage: The first controlled study from the early 1960 s by McKissock $^{25}$ compared hematoma evacuation to conservative management when no benefit from surgery was noted in regard to mortality or morbidity. The surgical trial in intracerebral hemorrhage (STICH $)^{26}$ was the first well-powered multicenter multinational RCT (1,033 patients) to compare the benefits of early hematoma drainage with initial conservative management. No overall benefit in functional outcome was found with early hematoma drainage and the mortality rate was similar in both groups. Further subgroup analysis including age, hematoma volume, hemorrhage location, anticoagulation- or thrombolytic-associated hemorrhage, severity of neurological deficit, type of intended operation, hematoma side, depth from the cortical surface, and country showed no benefit of early surgery across all subgroups except for a possible benefit in the patients with superficial hematoma. This led to the STICH II ${ }^{27}$ trial (601 patients) with superficial hematomas within $1 \mathrm{~cm}$ from the cortical surface. No overall benefit in functional outcome or mortality benefit was detected. The STICH trials were combined in a meta-analysis with 13 other studies when potential survival benefit in the intervention group was difficult to analyze as multiple surgical strategies, like craniotomy, endoscopic surgery, and stereotactic with/without plasminogen activator, limited the validity of the meta-analysis. Thus, the STICH RCTs did not show functional outcome or mortality benefit with early hematoma evacuation, particularly in deep hemorrhages and in small lobar hemorrhages with preserved level of consciousness. In large hematomas with mass effect and midline shift leading to altered levels of consciousness or when delayed neurological deterioration occurs through hematoma expansion as an important lifesaving measure, craniotomy and hematoma drainage are recommended. Ideal patient selection criteria for hematoma evacuation needs further determination.
Third, minimally invasive surgical techniques: Since the first trial of minimally invasive surgery in the 1980s comparing the use of endoscopic hematoma evacuation with conservative management using neuro endoscopy by Auer et $\mathrm{al}^{28}$ (which showed a lower mortality and higher rate of favorable outcome after 6 months in patients with subcortical hemorrhages who were alert and somnolent but not in patients who were stuperose/comatose, and neither in putaminal nor thalamic hemorrhages), the recent ICES-intraoperative computed tomography guided endoscopic surgery for brain hemorrhage-trial ${ }^{29}$ tested effectiveness of computed tomography (CT)-guided endoscopic drainage of ICH. The study was not powered to assess functional outcome and mortality, although compared with the medical group from the MISTIE-minimally invasive catheter evacuation followed by thrombolysis-trial (see below), the surgical group of ICES trial showed a nonsignificant favorable neurological outcome on mRS at 12 months.

Stereotactic surgery: In the MISTIE trials, ${ }^{30}$ further data and experience were obtained for surgical management of ICH by stereotactic or image-guided placements with thrombolysis and clot evacuation. In the phase 2 MISTIE study performed in 26 centers across North America and Europe, adults with spontaneous ICH and hematoma volume more than $20 \mathrm{~mL}$ were allocated to conservative management or MISTIE and tPA with the goal of a clot size reduction to less than $15 \mathrm{~mL}$. In this phase 2 study, accurate and safe drainage of the ICH was established followed by serial thrombolysis through a stereotactically targeted catheter that led to the phase 3 study. The MISTIE-III ${ }^{31}$ trial performed at 78 hospitals in North America, Europe, Australia, and Asia involving 506 patients (255 MISTIE group versus 251 for conservative management) measured an mRS score of 0 to 3 at 12 months. Despite a significant reduction in hematoma size, no outcome benefit was found. Adverse events were similar in the two groups. Thus, the MISTIE technique confirmed safety although did not improve long-term functional outcome. $^{32,33}$

The SCUBA-stereotactic intracerebral hemorrhage underwater blood aspiration ${ }^{34}$-technique performed in 47 patients in two phases-the first phase under dry field conditions and the second using a wet field strategy, where the surgeon is able to see the residual clot during hematoma drainage facilitating cauterization of possible bleeding vessels-has not been compared with other existing approaches.

Other ongoing RCTs-early minimally invasive removal of ICH, minimally invasive endoscopic surgical treatment with Apollo/Artemis in patients with brain hemorrhage, and a prospective multicenter study of Artemis in minimally invasive neuro evacuation device-use different strategies for both patient inclusion criteria and evacuation methodology. ${ }^{35}$

Fourth, patients in coma with GCS score less than 8, midline shift, and large hematomas or patients with refractory ICP based on only class III evidence showed that decompressive craniectomy with or without hematoma evacuation had a better outcome. In the study by Fung et al, ${ }^{36}$ decompressive craniectomy without hematoma evacuation in supratentorial ICH showed less mortality and better outcome at 6 months 
compared with the control group. The use of decompressive craniectomy with hematoma drainage was compared with hematoma drainage by craniotomy. Decompressive craniectomy in putaminal hemorrhage was associated with a significant improvement in midline shift and a trend toward better outcome. In the subgroup of patients with lobar ICH, decompressive craniectomy did not reveal a benefit. ${ }^{37}$

Fifth, posterior fossa hemorrhage: In $\sim 5$ to $13 \%$ of all ICH cases, a severe life-threatening bleeding occurs in the cerebellum or brainstem..$^{38}$ Due to the life-threatening nature of this condition, no randomized controlled clinical trial comparing early surgical evacuation with/without occipital decompressive craniectomy versus conservative management is available or likely possible. Only class III evidence is therefore available for suboccipital decompressive craniectomy, EVD insertion for hydrocephalus, or conservative management. These suggest cerebellar hemorrhage greater than $3 \mathrm{~cm}$ in diameter; cerebellar hemorrhage compressing the brainstem or causing acute hydrocephalus may be better managed with early ("early" not well defined) surgery. Patients with preserved levels of consciousness with cerebellar hematomas may be initially managed conservatively with urgent suboccipital craniectomy with/without hematoma drainage for acute neurological deterioration (GCS score $\leq 13$ ). ${ }^{39,40}$ In a multicenter retrospective study in 22 Italian hospitals, mortality was $38 \%$ for cerebellar hematomas versus $57 \%$ for brainstem hematomas ( 155 cerebellar and 50 brainstem hematomas). Level of consciousness 3 hours after initial hemorrhage and size of hemorrhage less than $3 \mathrm{~cm}$ were associated with better outcome. In brainstem hemorrhage, initial loss of consciousness and hematoma size were the main outcome determinants irrespective of hydrocephalus. This group proposed medical treatment for brainstem hematomas, and for larger lesions greater than $1.8 \mathrm{~cm}$ the outcome was uniformly fatal.

Kirollos et $\mathrm{a}^{38}$ developed a grading system based on the fourth ventricle size, configuration and location found in the CT scan. With GCS score greater than 13 and normal or compressed/distorted fourth ventricle, conservative management was proposed. With neurological deterioration and evolving hydrocephalus, EVD insertion followed by hematoma evacuation was advised. With complete effacement of the fourth ventricle, hematoma evacuation and CSF drainage are recommended.

Kuramatsu et $\mathrm{al}^{41}$ evaluated functional outcome of evacuation of cerebellar hematomas. In this meta-analysis of 4 observational ICH studies in 64 hospitals in the USA and Germany. The primary outcome was the proportion of patients with favorable outcome (mRS $=0-3)$ at 3 months. Secondary outcomes included the following: survival at 3 months, dichotomized functional outcome ( $\mathrm{mRS}=0-3 \mathrm{vs}$ 4-6) at 12 months, and survival at 12 months. Hematoma evacuation was not associated with better functional outcome at 3 months although hematoma evacuation was significantly associated with improved survival at 3 and 12 months. The surgical evacuation of hematomas less than $12 \mathrm{~mL}$ was found to be harmful while the evacuation of hematomas more than $15 \mathrm{~mL}$ was associated with improved survival without a beneficial effect on functional outcome.

\section{International Guideline Recommendations}

American Heart Association/American Stroke Association guidelines $^{42}$ for the management of spontaneous ICH and the European Stroke Organization ${ }^{43}$ guidelines for spontaneous ICH recommend for the majority of patients that with spontaneous supratentorial hemorrhage the benefit of surgical evacuation is not well established (class IIb; level of evidence A) ${ }^{42}$ with no supporting evidence for routine surgery (moderate quality, weak recommendation). ${ }^{42}$ For patients with a GCS score of 9-12, surgery may be lifesaving (moderate quality, weak recommendation) as well as for patients with delayed neurological deterioration (class IIb; level of evidence C). ${ }^{42}$

Decompressive craniectomy with/without hematoma evacuation may reduce mortality in patients with putaminal ICH, especially in comatose patients with large hematomas leading to significant midline shift and in patients with refractory intracranial hypertension (class IIb; level of evidence C). ${ }^{42}$

The effectiveness of the use of minimally invasive surgical approaches, such as stereotactic or endoscopic aspiration with or without thrombolysis, remains uncertain (class IIb; level of evidence B). ${ }^{42}$

In patients with posterior fossa hemorrhage with acute hydrocephalus, brainstem compression, or worsening in neurological status, surgery is to be performed as soon as feasible (class I; level of evidence B). ${ }^{42}$

\section{Downsides of Surgical ICH Trials (So Far)}

Neurosurgical patients requiring urgent procedures are difficult to recruit and the ideal candidate and the optimal timing of surgery have not been determined. ${ }^{44}$ The perspective of clinicians considering hematoma drainage as a lifesaving measure comes in the way of randomization of these patients in these interventional studies. There is a significant crossover from medical management to the surgical arm of these studies, thereby concealing the otherwise higher rates of an unfavorable outcome and death in the conservative management arm. Problems of study design, sample size, and number of excluded patients affect conclusions. Very restrictive inclusion protocols ${ }^{45}$ have resulted in slow recruitment as evidenced by only $9.5 \%$ of lobar ICH without IVH and only $3.7 \%$ of all ICH patients meeting inclusion criteria in the STICH II trial, a population-based study. These restrictions may have limited our understanding so far on the evidence base for spontaneous ICH surgical intervention procedures. We should endeavor to address the above, gather further evidence, and change management/surgical practice wherever necessary for the benefit of the patient.

\section{Conflict of Interest}

None declared.

\section{References}

1 van Asch CJ, Luitse MJ, Rinkel GJ, van der Tweel I, Algra A, Klijn CJ. Incidence, case fatality, and functional outcome of intracerebral haemorrhage over time, according to age, sex, 
and ethnic origin: a systematic review and meta-analysis. Lancet Neurol 2010;9(2):167-176

2 Ariesen MJ, Claus SP, Rinkel GJE, Algra A. Risk factors for intracerebral hemorrhage in the general population: a systematic review. Stroke 2003;34(8):2060-2065

3 Yamada M. Cerebral amyloid angiopathy: emerging concepts. J Stroke 2015;17(1):17-30

4 Kuramatsu JB, Sembill JA, Huttner HB. Reversal of oral anticoagulation in patients with acute intracerebral hemorrhage. Crit Care 2019;23(1):206

5 Frontera JA, Lewin JJ II, Rabinstein AA, et al. Guideline for reversal of antithrombotics in intracranial hemorrhage. Neurocrit Care 2016;24(1):6-46

6 Qureshi AI, Palesch YY, Barsan WG, et al; ATACH-2 Trial Investigators and the Neurological Emergency Treatment Trials Network. Intensive blood-pressure lowering in patients with acute cerebral hemorrhage. N Engl J Med 2016;375(11):1033-1043

7 Sprigg N, Flaherty K, Appleton JP, et al; TICH-2 Investigators. Tranexamic acid for hyperacute primary IntraCerebral Haemorrhage (TICH-2): an international randomised, placebocontrolled, phase 3 superiority trial. Lancet 2018;391(10135): 2107-2115

8 Mayer SA, Brun NC, Begtrup K, et al; FAST Trial Investigators. Efficacy and safety of recombinant activated factor VII for acute intracerebral hemorrhage. N Engl J Med 2008; 358(20):2127-2137

9 Wong JM, Ziewacz JE, Ho AL, et al. Patterns in neurosurgical adverse events: open cerebrovascular neurosurgery. Neurosurg Focus 2012;33(5):E15

10 Xi G, Keep RF, Hoff JT. Mechanisms of brain injury after intracerebral haemorrhage. Lancet Neurol 2006;5(1):53-63

11 Anderson CS, Heeley E, Huang Y, et al; INTERACT2 Investigators. Rapid blood-pressure lowering in patients with acute intracerebral hemorrhage. N Engl J Med 2013;368(25):2355-2365

12 Moullaali TJ, Wang X, Martin RH, et al. Blood pressure control and clinical outcomes in acute intracerebral haemorrhage: a preplanned pooled analysis of individual participant data. Lancet Neurol 2019;18(9):857-864

13 Broderick JP, Brott TG, Duldner JE, Tomsick T, Huster G. Volume of intracerebral hemorrhage. A powerful and easy-to-use predictor of 30-day mortality. Stroke 1993;24(7):987-993

14 Hemphill JC III, Bonovich DC, Besmertis L, Manley GT, Johnston SC, Tuhrim S. The ICH score: a simple, reliable grading scale for intracerebral hemorrhage. Stroke 2001;32(4):891-897

15 Witsch J, Neugebauer H, Zweckberger K, Jüttler E. Primary cerebellar haemorrhage: complications, treatment and outcome. Clin Neurol Neurosurg 2013;115(7):863-869

16 de Oliveira Manoel AL, Goffi A, Zampieri FG, et al. The critical care management of spontaneous intracranial hemorrhage: a contemporary review. Crit Care 2016;20:27210.1186/ s13054-016-1432-0

17 Gong C, Boulis N, Qian J, Turner DE, Hoff JT, Keep RF. Intracerebral hemorrhage-induced neuronal death. Neurosurgery 2001;48(4):875-882, discussion 882-883

18 Qureshi AI, Ling GS, Khan J, et al. Quantitative analysis of injured, necrotic, and apoptotic cells in a new experimental model of intracerebral hemorrhage. Crit Care Med 2001;29(1):152-157

19 Hallevi H, Albright KC, Aronowski J, et al. Intraventricular hemorrhage: anatomic relationships and clinical implications. Neurology 2008;70(11):848-852

20 Claude Hemphill J III, Lam A. Emergency neurological life support: intracerebral hemorrhage. Neurocrit Care 2017; 27(Suppl 1):89-101

21 Hanley DF, Lane K, McBee N, et al; CLEAR III Investigators. Thrombolytic removal of intraventricular haemorrhage in treatment of severe stroke: results of the randomised, multicentre, multiregion, placebo-controlled CLEAR III trial. Lancet 2017;389(10069):603-611
22 Hinson HE, Melnychuk E, Muschelli J, Hanley DF, Awad IA, Ziai WC. Drainage efficiency with dual versus single catheters in severe intraventricular hemorrhage. Neurocrit Care 2012;16(3):399-405

23 Staykov D, Kuramatsu JB, Bardutzky J, et al. Efficacy and safety of combined intraventricular fibrinolysis with lumbar drainage for prevention of permanent shunt dependency after intracerebral hemorrhage with severe ventricular involvement: a randomized trial and individual patient data meta-analysis. Ann Neurol 2017;81(1):93-103

24 Li Y, Zhang H, Wang X, et al. Neuroendoscopic surgery versus external ventricular drainage alone or with intraventricular fibrinolysis for intraventricular hemorrhage secondary to spontaneous supratentorial hemorrhage: a systematic review and meta-analysis. PLoS One 2013;8(11):e8059910.1371/journal.pone.0080599

25 Mckissock W, Richardson A, Taylor J. Primary intracerebral haemorrhage. Lancet 1961;278:221-226

26 Mendelow AD, Gregson BA, Fernandes HM, et al; STICH investigators. Early surgery versus initial conservative treatment in patients with spontaneous supratentorial intracerebral haematomas in the International Surgical Trial in Intracerebral Haemorrhage (STICH): a randomised trial. Lancet 2005;365(9457) : 387-397

27 Mendelow AD, Gregson BA, Rowan EN, Murray GD, Gholkar A, Mitchell PM; STICH II Investigators. Early surgery versus initial conservative treatment in patients with spontaneous supratentorial lobar intracerebral haematomas (STICH II): a randomised trial. Lancet 2013;382(9890):397-408

28 Auer LM, DeinsbergerW, Niederkorn K, etal.Endoscopic surgery versus medical treatment for spontaneous intracerebral hematoma: a randomized study. J Neurosurg 1989;70(4):530-535

29 Vespa P, Hanley D, Betz J, et al; ICES Investigators. ICES (intraoperative stereotactic computed tomography-guided endoscopic surgery) for brain hemorrhage: a multicenter randomized controlled trial. Stroke 2016;47(11):2749-2755

30 Hanley DF, Thompson RE, Muschelli J, et al; MISTIE Investigators. Safety and efficacy of minimally invasive surgery plus alteplase in intracerebral haemorrhage evacuation (MISTIE): a randomised, controlled, open-label, phase 2 trial. Lancet Neurol 2016;15(12):1228-1237

31 Hanley DF, Thompson RE, Rosenblum M, et al; MISTIE III Investigators. Efficacy and safety of minimally invasive surgery with thrombolysis in intracerebral haemorrhage evacuation (MISTIE III): a randomised, controlled, open-label, blinded endpoint phase 3 trial. Lancet 2019;393(10175):1021-1032

32 Awad IA, Polster SP, Carrión-Penagos J, et al; MISTIE III Trial Investigators. Surgical performance determines functional outcome benefit in the minimally invasive surgery plus recombinant tissue plasminogen activator for Intracerebral hemorrhage evacuation (MISTIE) procedure. Neurosurgery 2019;84(6): 1157-1168

33 Mould WA, Carhuapoma JR, Muschelli J, et al; MISTIE Investigators. Minimally invasive surgery plus recombinant tissue-type plasminogen activator for intracerebral hemorrhage evacuation decreases perihematomal edema. Stroke 2013;44(3): 627-634

34 Kellner CP, Chartrain AG, Nistal DA, et al. The Stereotactic Intracerebral Hemorrhage Underwater Blood Aspiration (SCUBA) technique for minimally invasive endoscopic intracerebral hemorrhage evacuation. J Neurointerv Surg 2018;10(8): 771-776

35 Scaggiante J, Zhang X, Mocco J, Kellner CP. Minimally invasive surgery for Intracerebral hemorrhage. Stroke 2018;49(11):2612-2620

36 Fung C, Murek M, Z'Graggen WJ, et al. Decompressive hemicraniectomy in patients with supratentorial intracerebral hemorrhage. Stroke 2012;43(12):3207-3211 
37 Hayes SB, Benveniste RJ, Morcos JJ, Aziz-Sultan MA, Elhammady MS. Retrospective comparison of craniotomy and decompressive craniectomy for surgical evacuation of nontraumatic, supratentorial intracerebral hemorrhage. Neurosurg Focus 2013;34(5):E3

38 Kirollos RW, Tyagi AK, Ross SA, van Hille PT, Marks PV. Management of spontaneous cerebellar hematomas: a prospective treatment protocol. Neurosurgery 2001;49(6):1378-1386, discussion 1386-1387

39 Mathew P, Teasdale G, Bannan A, Oluoch-Olunya D. Neurosurgical management of cerebellar haematoma and infarct. J Neurol Neurosurg Psychiatry 1995;59(3):287-292

40 Kobayashi S, Sato A, Kageyama Y, Nakamura H, Watanabe Y, Yamaura A. Treatment of hypertensive cerebellar hemorrhagesurgical or conservative management? Neurosurgery 1994; 34(2):246-250, discussion 250-251

41 Kuramatsu JB, Biffi A, Gerner ST, et al. Association of Surgical Hematoma Evacuation vs conservative treatment with functional outcome in patients with cerebellar Intracerebral hemorrhage. JAMA 2019;322(14):1392-1403
42 Hemphill JC II, Greenberg SM, Anderson CS, et al; American Heart Association Stroke Council; Council on Cardiovascular and Stroke Nursing; Council on Clinical Cardiology. Guidelines for the management of spontaneous intracerebral hemorrhage: a guideline for healthcare professionals from the American Heart Association/American Stroke Association. Stroke 2015;46(7):2032-2060

43 Steiner T, Al-Shahi Salman R, Beer R, et al; European Stroke Organisation. European Stroke Organisation (ESO) guidelines for the management of spontaneous intracerebral hemorrhage. Int J Stroke 2014;9(7):840-855

44 Rabinstein AA, Wijdicks EFM. Surgery for intracerebral hematoma: the search for the elusive right candidate. Rev Neurol Dis 2006;3(4):163-172

45 Kirkman MA, Greenwood N, Singh N, Tyrrell PJ, King AT, Patel HC. Difficulties with recruiting into neurosurgical clinical trials: the Surgical Trial in IntraCerebral Haemorrhage II as an example. Br J Neurosurg 2011;25(2):231-234 